
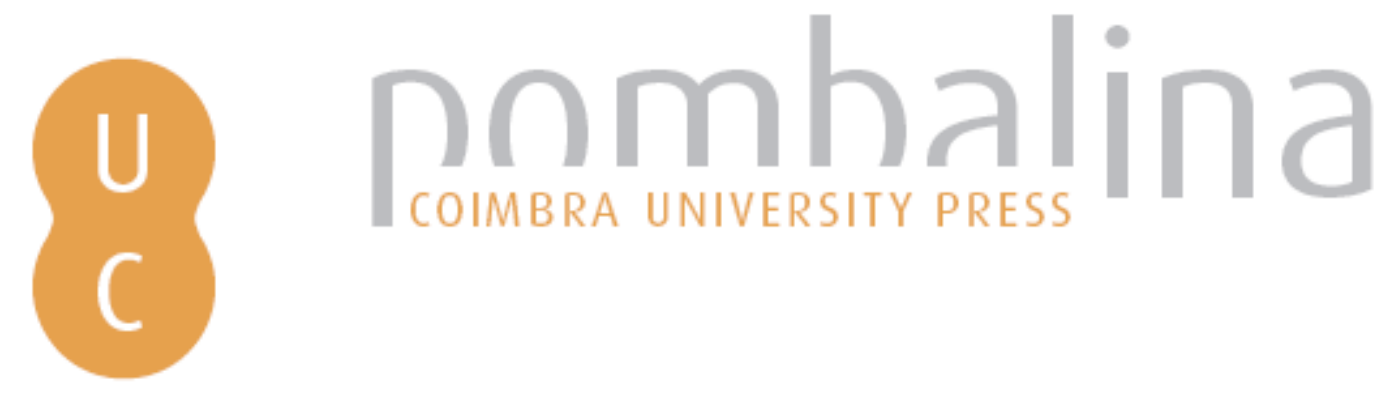

Después de la destrucción del estado y del fracaso del mercado: el redescubrimiento de la sociedad, su conversión en "capital" y su papel en la legitimación de la desigualdad
Autor(es):
Marrero, Adriana

Publicado por: Imprensa da Universidade de Coimbra

URL persistente:

URl:http://hdl.handle.net/10316.2/31322

DOI:

DOI:http://dx.doi.org/10.14195/978-989-26-0222-6_5

Accessed : $\quad$ 26-Apr-2023 09:07:29

A navegação consulta e descarregamento dos títulos inseridos nas Bibliotecas Digitais UC Digitalis, UC Pombalina e UC Impactum, pressupõem a aceitação plena e sem reservas dos Termos e Condições de Uso destas Bibliotecas Digitais, disponíveis em https://digitalis.uc.pt/pt-pt/termos.

Conforme exposto nos referidos Termos e Condições de Uso, o descarregamento de títulos de acesso restrito requer uma licença válida de autorização devendo o utilizador aceder ao(s) documento(s) a partir de um endereço de IP da instituição detentora da supramencionada licença.

Ao utilizador é apenas permitido o descarregamento para uso pessoal, pelo que o emprego do(s) título(s) descarregado(s) para outro fim, designadamente comercial, carece de autorização do respetivo autor ou editor da obra.

Na medida em que todas as obras da UC Digitalis se encontram protegidas pelo Código do Direito de Autor e Direitos Conexos e demais legislação aplicável, toda a cópia, parcial ou total, deste documento, nos casos em que é legalmente admitida, deverá conter ou fazer-se acompanhar por este aviso.

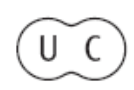


Sílvia Portugal

Paulo Henrique Martins

Organização

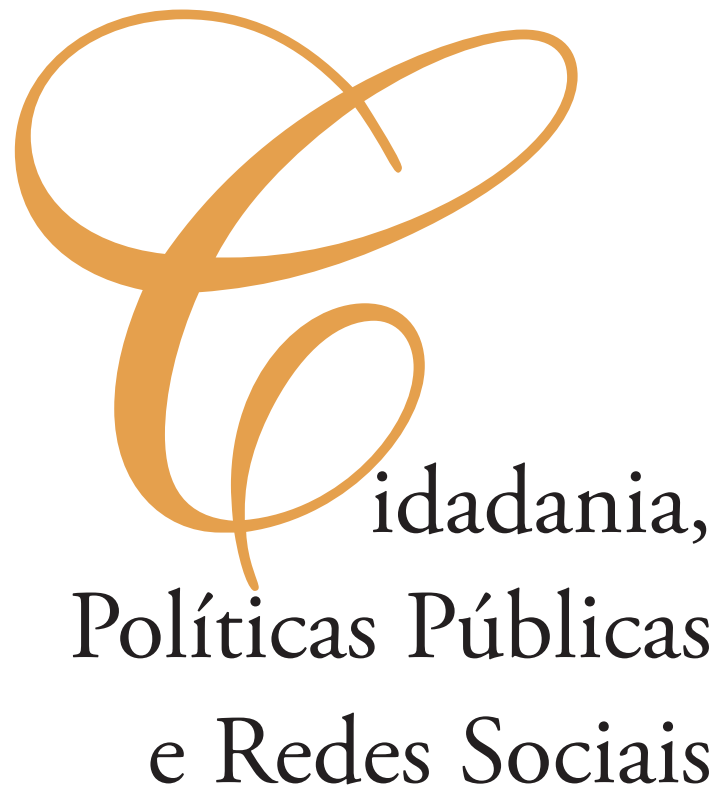


Adriana Marrero

\section{DESPUÉS DE LA DESTRUCCión DEL ESTADO Y DEL FRACASO DEL MERCADO. EL REDESCUBRIMIENTO DE LA SOCIEDAD, SU CONVERSIÓN EN “CAPITAL” Y SU PAPEL EN LA LEGITIMACIÓN DE LA DESIGUALDAD}

\section{Introducción}

Tal vez uno de los aspectos más llamativos de la teoría social actual, es el aparente re-descubrimiento de la sociabilidad. Más curioso aún, es que este "re-descubrimiento" está siendo llevado a cabo, principalmente, desde la propia Sociología. Al fin de cuentas, ¿qué es la Sociología, sino el estudio de las relaciones sociales? ¿Qué es si no el estudio de la sociabilidad? Son muchos y variados los factores que nos han traído a este ya viejo lugar de reflexión y de análisis; algunos de ellos serán examinados luego. Pero lo cierto es que un siglo y medio después de la aparición de las primeras construcciones teóricas que guiaron el desarrollo de los distintos paradigmas que han conformado a la Sociología como ciencia, nos encontramos, nuevamente, ante la circunstancia de discutir la sociabilidad y preguntarnos sobre su naturaleza y dinámica.

Variados son también los nuevos conceptos que recientemente han dado cobijo a estas nuevas interrogantes sobre la sociabilidad: el "capital social" es uno de ellos y como tal ha venido a describir las características y los efectos que tiene la sociabilidad humana y el agrupamiento de personas en su bienestar físico, anímico, y social. Pero como toda teoría, ésta también tiene su origen en intereses y en perspectivas prácticas, y no está ajena a la necesidad de pensar la realidad social e imaginar nuevas maneras de diseńo y puesta en práctica de políticas públicas destinadas a combatir la pobreza y la marginalidad, principalmente en el mundo no desarrollado.

En este artículo me propongo mostrar algunos supuestos básicos de la teoría en algunas de sus vertientes, mostrar algunos aspectos críticos, y proponer algunas hipótesis que pueden explicar su éxito en las ciencias políticas y sociológicas de los últimos lustros.

\section{El redescubrimiento y su contexto}

El auge liberalizador de los ochenta tuvo profundos y devastadores efectos en el tejido social de los países no desarrollados. Las crisis económicas de los noventa, y en el cono sur de América Latina, de principios del este siglo, pusieron en evidencia la desprotección de vastos sectores sociales que, sin el auxilio de la red de políticas sociales que habían sido 
desmanteladas con el achicamiento del estado como resultado de la aplicación de políticas liberales, cayeron rápida y masivamente por debajo de la línea de pobreza y aún, de la indigencia. Este era un panorama inédito para unos países tradicionalmente definidos como de "capas medias", desacostumbrados al hambre y a la privación. El modelo debía ser revisado. Al final de cuentas, el mercado no podía resolverlo todo, de la mejor manera. Pero por otro lado, el fracaso neoliberal no convenció a los impulsores del modelo de la necesidad de revisar su desconfianza en relación al estado. En la opinión pública, la prolongada prédica antiestatista había surtido efecto, y pocos esperaron ya que se volviera a apelar a la acción integradora del estado y de su institucionalidad para el diseño y aplicación de políticas sociales. Se generaron así las condiciones para el surgimiento de una nueva teoría que sirviera como inspiradora de políticas públicas, al solucionar un doble problema: la identificación de un nuevo factor explicativo de la crisis social y su uso como factor clave para el desarrollo. Ese concepto fue el de "capital social".

El capital social enfocó los microprocesos de solidaridad recíproca al interior de los pequeños grupos sociales, constituyéndose en una "tercera vía" entre el estado y el mercado, permitiendo la visualización de unas formas de intercambio económico que quedaban frecuentemente invisibilizadas debido, en parte, al carácter no monetario de los intercambios. El trueque, la donación, y las ayudas en formas de servicios o regalos desinteresados entre vecinos, amigos y parientes, comenzaron a ser vistos no ya sólo como una red de solidaridad apoyada en la afectividad y en el compromiso moral que sostiene a los pobres -la única en momentos de crisis extrema- sino como una forma encubierta de circulación económica que no podía quedar fuera de la "contabilidad" oficial.

Recordemos que la sociología no desconocía la existencia de esas relaciones informales y su utilidad para el sostén vital y emocional de las personas y la teorización sobre la importancia de la interdependencia recíproca en comunidades tiene una larga tradición ${ }^{1}$.

Desde que Tönnies formulara su clásica distinción entre Gemeinschaft y Gesellschaft, estos conceptos sufrirían sucesivas reinterpretaciones en los diversos esquemas teóricos, pero siempre conservando lo que constituye el núcleo duro de la polaridad: el que señala la oposición entre una lógica racional, formal e impersonal - que fundamenta la economía clásica y la organización burocrática (Gesellschaft), y la motivación afectiva, según la cual las redes y agrupaciones que forman los sujetos involucrados constituyen, para ellos, fines en sí mismos (Gemeinschaft). En términos simples, la Gemeinschaft se basa en vínculos de sangre o parentesco, de lugar o vecindad y de afecto o amistad, y se reconoce por los siguientes atributos: unidad, división del trabajo basada en la ayuda y asistencia mutua; equilibrio de las voluntades humanas en interdependencia mutua; autoridad basada en la edad y la sabiduría, un hábitat común, acción común dirigida hacia objetivos comunes que se consideran dados; relaciones de parentesco; relaciones de amistad; sentimiento recíproco y obligatorio; obligaciones difusas y generales; idioma, costumbres y creencias comunes; posesión y diversiones mutuas; espíritu de hermandad. La Gesellschaft se basa en la voluntad racional y consiste en relaciones contractual y funcionalmente específicas, establecidas conscientemente para el alcance de objetivos planteados. Está articulada en medio de la convención, la legislación y la opinión pública, y se distingue por los siguientes atributos: individualismo, acción en términos del propio interés; reglamentaciones positivas

1 Una revisión amplia y comprensiva del concepto de "redes sociales" de las distintas perspectivas teóricas relacionadas con el mismo puede encontrarse en Portugal (2007). 
y específicas; esferas de contacto delimitadas; relaciones de dinero y crédito; obligaciones limitadas; falta de relaciones familiares mutuas. Tönnies sostenía que la evolución histórica transcurría desde la Gemeinschaft a la Gesellschaft, aunque en los hechos ambos tipos pueden coexistir y encontrarse interrelacionados, en grados diversos, en distintas estructuras empíricas (Mc. Kinney, 1968: 118-119). Desde aquí es posible rastrear la tipología a través de casi toda la teoría social.

Sin embargo, también es cierto que a pesar del innegable lugar asignado a los lazos afectivos y comunitarios en toda a teoría social, la sociología moderna estuvo dominada por la supremacía de la racionalidad en todas sus formas. Al identificar las relaciones sociales familiares y corporativas a nivel micro, eminentemente particularistas, con formas premodernas, irracionales, o relativamente primitivas de estructuración social (como la tribu, el feudo, los gremios medievales o la aristocracia), la teoría termina atribuyendo a estos lazos una posición subalterna a los vínculos universalistas, formales y racionales, en los que se fundaba el orden social moderno y sus instituciones. A nivel político-práctico, los esfuerzos de construcción institucional de organizaciones modernas y racionales, tales como el Estado o la Economía - más o menos planificada, más o menos liberal - contribuyó al progresivo olvido de los efectos económicos del relacionamiento comunitario.

Esto es, en parte, lo que redescubre la teoría del Capital Social (CS). En otros lugares (Marrero, 2004, 2006), he mostrado la teoría misma y he desarrollado argumentos críticos sobre ella, tanto desde el punto de vista de su lógica teórica, como desde la consideración de sus consecuencias prácticas. No volveré sobre ello. Pero baste con señalar que las actuales formulaciones de la teoría - que son variadas y heterogéneas - pueden ser agrupadas en dos distintas vertientes.

a) Por un lado, están aquellas que ven el CS - como forma de sociabilidad - y a las transacciones que ocurren dentro de la familia, el barrio o el micro grupo, dentro de la dinámica más general de la estructuración y la reproducción sociales. El principal exponente de esta visión es, por supuesto, Pierre Bourdieu. Expresada en términos simples, esta visión del CS se basa en el supuesto de que la sociedad se reproduce y se perpetúa a sí misma, a través de los esfuerzos que desarrollan las personas de los distintos grupos por mantener y trasmitir a sus descendientes aquellos bienes sociales que son característicos de su lugar en la sociedad, lo que a nivel macro se expresa a través de la permanencia de las diferenciaciones, desigualdades, y distancias sociales que separan a unos grupos de otros.

Como en las sociedades modernas el ordenamiento social se encuentra explícitamente y jurídicamente legitimado por el principio del logro y del mérito, estos actos de transmisión de bienes y privilegios sociales deben asumir un carácter relativamente oculto, para lo cual suelen ser eufemizados o disimulados a través de mecanismos diversos, entre los cuales encuentra un lugar destacado la transformación de unas formas de capital en otras: el capital económico se transforma a través de inversiones en el cultivo de relaciones apropiadas y en una escolarización determinada, en CS y educativo, los que rendirán unos beneficios que asegurarán la permanencia y la transmisión de capital - de nuevo económico - a la siguiente generación. En esta perspectiva, el CS no es más que una forma del capital y por lo tanto no es independiente de éste. Poseen CS los poseedores de capital. En esta perspectiva crítica, por tanto, el CS es una forma más de ocultamiento de la apropiación del beneficio social por parte de unas clases en perjuicio de otras (Bourdieu).

b) Por otro lado, se encuentran aquellas teorías que ven al CS como una forma cualquiera de obtener beneficios de cualquier tipo, a través del contacto continuado en el tiempo 
con otras personas del mismo grupo social. James Coleman o Francis Fukuyama son exponentes clásicos de esta vertiente. Desde este punto de vista, el CS se aproxima al concepto de "densidad moral" de Durkheim, en el sentido de que cuanto más numerosas sean las relaciones sociales que pueda mantener una persona, más rico será en términos de CS. Las relaciones con los propios familiares, vecinos del barrio, conocidos en general, le permiten a la persona acceder a través de intercambios de favores, expectativas de reciprocidad futura y otros mecanismos semejantes, a los beneficios que resultan del acceso a la disponibilidad de otros. Desde esta perspectiva, el CS, a diferencia de las otras formas de capital, puede encontrarse en personas de cualquier clase, sea cual sea su lugar social. Los pobres o indigentes que logran satisfacer ocasionalmente sus necesidades más básicas de alimento gracias a la solidaridad de otros, contarán con CS siempre que dispongan de estos "otros" a quienes recurrir. El capital, entonces, puede estar presente a todo lo largo de la escala social. Asimismo, las relaciones aparentemente más desinteresadas, como la de una madre con su hija o hijo, las relaciones de pareja o de cualquier otro tipo, pueden ser importantes fuentes de CS. En otras versiones de este mismo abordaje, - típicamente en la de Robert Putnam - el CS asume la forma de normas institucionalizadas que dotan de previsibilidad a la acción individual: la vigencia del ordenamiento jurídico, la efectividad de la acción estatal o el respeto de las normas de tránsito, pueden acrecentar el CS de las personas que pertenecen a la comunidad donde se aplica dicho ordenamiento. Estas últimas formulaciones, despojadas del carácter crítico que la teoría de Bourdieu asignaba al concepto de "capital", son las que han venido predominando en la producción sociológica sobre el tema.

Como puede desprenderse de acá, entonces, la principal contribución de la teoría del CS consiste no en el descubrimiento de tales lazos en sí mismos para la conformación de un orden moral de reciprocidad (como ya había hecho Durkheim), ni en su importancia en términos de generación de riqueza y de eficacia histórica (como hizo Weber), sino en su revalorización como locus de reencuentro y de reconciliación de lo comunitario, familiar y privado con lo asociativo, instrumental, y público como generadores de riqueza social, a través de la simple atribución o la acentuación de un carácter económico a intercambios que tienen muchas veces - ante los ojos de sus participantes - una naturaleza distinta.

Buena parte de las objeciones a la teoría que hemos formulado en otros lugares, no tiene que ver entonces con el re-descubrimiento de la importancia de la sociabilidad, lo que es hasta cierto punto banal. De lo que se trata, es de mostrar cómo un fenómeno que es constitutivo de la dinámica de funcionamiento y conservación de las sociedades - incluso de reproducción de las desigualdades más notorias de esas sociedades - son elevados a la categoría de factores francamente positivos, y potenciadores del desarrollo de las sociedades.

\section{El éxito de la teoría}

Son varios los factores que explican que este concepto, impulsado principalmente desde los "think tanks", haya tenido una recepción tan favorable entre los diseñadores de políticas a nivel global, los gobiernos nacionales, los actores locales, las organizaciones no gubernamentales, y gran parte de la teoría social. Algunos de ellos pueden ser:

1. Se trata de un enfoque que se centra en un aspecto ya conocido de la vida social -los lazos de solidaridad basados en la confianza que proporciona la pertenencia a grupos- al 
que se atribuye un rendimiento económico. Esta teorización fue el "toque de Midas" para unas relaciones que, desde el auge de la modernidad y de la asociatividad como modelo racionalizador de las relaciones sociales, había relegado la sociabilidad cotidiana al ámbito subordinado de lo privado, lo emotivo, lo simple, lo primitivo, lo no desarrollado, lo irracional. Todo aquello que, bajo el peso simbólico de la hegemonía de las racionalidades del igualitarismo del estado benefactor o del liberalismo del intercambio mercantil era visto como marginal, como no relevante, como opaco, o incluso, como resistente, se convirtió, como por encanto, en el cuerno de oro de la abundancia social.

2. A nivel de políticas públicas, esto abre posibilidades únicas para naciones que -hundidas en la pobreza y fracturadas socialmente- descubren que el secreto de la riqueza de sus países dormitaba en el seno de aquello en lo que eran "verdaderamente" ricas: masas de personas que lo único que tenían era a sus semejantes. No es sorprendente que muchos gobiernos, imposibilitados de acudir a un aparato estatal de bienestar social desmantelado por décadas de prédica anti-intervencionista, y con un sistema de mercado debilitado por la extensión de la pobreza extrema, la desigualdad, y las sucesivas crisis del capitalismo global, vieran con buenos ojos la posibilidad de contabilizar como parte de la riqueza nacional, el valor económico de unos intercambios familiares, comunitarios, vecinales que, limitadas al ámbito de lo particular, y realizados con otros propósitos y sobre otras bases, no encontraban lugar en el ámbito de lo público. El que en esos intercambios se transaran bienes y servicios de muy distinta calidad y cantidad, no evitó que se intentara ver en todos ellos, aún en los más miserables, los que apenas permitían una subsistencia elemental, una fuente y una muestra de la riqueza social.

3. Más aún: por esta inversión simbólica de los términos, no es difícil ver cómo aquellos sujetos que más frecuentemente se constituían en acreedores de la acción del estado al que responsabilizaban por su omisión como prestador de políticas sociales, pasaban - en algunas versiones de la teoría - a la condición de deudores, al ser responsabilizados por los efectos que su aislamiento social podía tener en su propia suerte y la de sus hijos. Recordemos que planteos como el de Fukuyama, pero también el de muchos otros, terminan por responsabilizar a los sectores más vulnerables - en especial a las mujeres jefas de hogar - por el perjuicio que, en términos de pérdida de CS, ocasiona a los hijos el alejamiento del hombre del hogar, como consecuencia del alza en la tasa de divorcios. Los más desfavorecidos, llegan así, a convertirse en una suerte de "deudores sociales" por no asegurar a su entorno inmediato afectivo - hijos y descendientes - una red de relaciones adecuada o favorable para el aprovechamiento de la estructura de oportunidades que la sociedad, en esta perspectiva, abriría a todos, prácticamente por igual. A nivel simbólico, el estado queda así, exonerado de la responsabilidad por el bienestar social de unos sujetos que han descuidado o abandonado la pertenencia a una estructura familiar que les habría favorecido económicamente, a sí mismos o a sus hijos.

4. A nivel de la acción comunitaria, muchas organizaciones no gubernamentales, comunidades religiosas, caudillos locales, y otros grupos de acción y promoción social, de diverso tipo, procedencia y propósitos, vieron de pronto cómo su actividad cobraba nueva relevancia, y cómo esta nueva gravitación en su práctica local era además apoyada, financiada y legitimada por el flujo a veces no menor de recursos que llegaban desde un estado 
que había perdido, abandonado o anulado los viejos canales de prestación de servicios. El traspaso a las ONGs de una parte de las funciones y prestaciones que antes eran servidas por el estado, fue un modo de traspasar servicios públicos al ámbito privado (esto es, de privatizar, (McDonald y Ruiters, 2006) evitando las resistencias que generan estos procesos cuando las funciones públicas son traspasadas a grandes empresas, nacionales o trasnacionales. Mientras, las organizaciones vecinales y locales, en particular aquellas de tipo religioso, ven en estas formas de financiamiento estatal a los servicios sociales que prestan, un subsidio indirecto a una labor pastoral que había permanecido eclipsada durante largo tiempo por el apogeo del estado laico de bienestar.

5. Esto tiene múltiples impactos en los modos de visualizar a este tipo de relaciones: a) les dota de un carácter económico como generador de riqueza en sentido estricto, lo que satisface a algunos economistas; b) las resignifica como un lugar clave para el desarrollo social de las naciones, revitalizando el interés en el estudio de la sociabilidad, lo que satisface a buena parte de la comunidad sociológica; c) las revaloriza al invertir la jerarquía instaurada por la modernidad entre comunidad y asociación, devolviendo a la primera una valoración ya muy maltrecha por el ascenso de las pretensiones universalistas modernas; esto es particularmente grato a las comunidades religiosas, de acción comunitaria, de promoción social, organizaciones no gubernamentales, y otros grupos de poder y liderazgo localmente arraigados, que no sólo ven legitimada su influencia social, sino muchas veces además, la ven fortalecida por el financiamiento de su actividad regular por parte del estado y de la cooperación internacional; d) las expone como mecanismos legítimos, no sólo de generación, sino de apropiación y transmisión de la riqueza, con lo cual, -al otro extremo de la escala social, en las élites- se justifica la circulación familiar del privilegio social y político, y el aprovechamiento de las redes particularistas y exclusivas, por donde circula el grueso del poder económico, político y social.

6. Se trata, en suma, de una teoría altamente polisémica y multifuncional, que da a cada uno lo que cada uno necesita oír, cuya virtud más evidente es la de conciliar los intereses y las visiones de todos aquellos que tienen voz: las élites políticas, económicas y sociales, las organizaciones no gubernamentales, y comunales, de tipo social y religioso, y hasta a las clases medias bien insertas socialmente, obvia y comprensiblemente ajenas a la polémica, pero renuentes a soportar mayores cargas fiscales para financiar la reconstrucción del estado de bienestar.

\section{3. ¿Qué falla en la Teoría del CS?}

En este apartado nos interesa señalar algunos grandes aspectos problemáticos de la teoría del CS, como es usada actualmente.

a) En primer término, si a ciertos resultados beneficiosos de la pertenencia a grupos queremos darle el nombre de "capital", debemos ser consecuentes con el concepto e incluirlos dentro de la lógica más general de la generación del capital y de su trasmisión. Desde nuestro punto de vista, no parece plausible sostener - con Coleman, Solow, Arrow, y muchos otros - que aunque adoptamos el nombre de capital, no se trata, realmente de capital ni responde a su lógica. $\mathrm{O}$ bien subsumimos el concepto bajo la lógica del capital, en cuyo caso el concepto 
encuentra una más cómoda ubicación en el esquema conceptual de Bourdieu - que postula la dependencia de unas formas de capital de otras, su transformación recíproca y su reductibilidad última a capital financiero, y que permite visibilizar las formas invisibles de trasmisión del capital que tienen lugar por su eufemización - o, por el contrario, abandonamos la noción de capital y la sustituimos por otra que designe de modo más adecuado aquello que los autores reconocen como rasgos característicos. En nuestra perspectiva, la aproximación bourdiana es la que cumple mejor con los propósitos de iluminar los rendimientos económicos que ciertas formas de sociabilidad tienen para los individuos, así como para la mostrar la determinación social de las diferencias en dichos rendimientos.

b) En segundo lugar, para designar todas aquellas formas de ayuda, solidaridad y cooperación que los sujetos aportan en una relación que es vista por los participantes como un fin en si misma, debería bastar con los aportes conceptuales provenientes de la sociología en el estudio de las distintas formas de acción social y de sus fundamentos, en las lineas de Giddens, Beck, Archer, Bauman, Habermas y tantos otros. Una buena sociología debería preocuparse por mantener las distinciones teórica y empírica entre las relaciones proveedoras de sentido, basadas en lazos de comunidad y afecto, de aquellas relaciones de intercambio racionalmente orientadas, por citar sólo dos de los varios tipos posibles. Sólo la obsesión contable por incrementar los activos societales de países en crisis, o el interés por superar la crítica al particularismo patrimonialista de ciertas prácticas políticas, pueden justificar que ambos tipos de relaciones hayan terminado, a pesar de su larga historia como tipos polares, confundidos bajo una sola, ambigua, etiqueta.

c) En tercer lugar, la teoría tiene un evidente sesgo economicista que atenta contra la comprensión de la variedad de las motivaciones que impulsan a los actores en su relacionamiento reciproco. Una sociología que tome en cuenta las interpretaciones que los propios actores hacen de sus acciones situadas, aunque, en una doble hermenéutica, las interprete a su vez, está obligada a tomar en consideración que la mayor parte de las acciones y relaciones que la teoría visualiza en términos de "CS", son experimentadas por los actores como acciones desinteresadas, afectivas, y contrarias a todo cálculo racional. En palabras de Eduardo Vizer:

"Encuadrar las normas y los valores sociales como la confianza y la participación dentro de la noción de capital parece ser - cuando menos - algo chocante y hasta cierto punto antagónico. (...) La propuesta de adopción de la noción de capital para encuadrar teóricamente un conjunto de procesos de organización social, relaciones, creencias y valores de pertenencia y compromiso colectivo, puede distorsionar el sentido y las características que la propia comunidad - y los actores involucrados - adjudican a estas formas "vividas" de la praxis social. Hay una construcción de "sentido" fundamental por parte de los actores y de una comunidad que debe formar parte del objetivo de cualquier proyecto de investigación sobre estos procesos sociales (...) El paradigma del CS, visualizado y objetivado como una forma de capital, corre en este sentido el riesgo de "cooptar" epistemológicamente el contenido social originario de las ideas propuestas como una ontología a ser empíricamente investigada" (Vizer, 2006:304-305)

La ampliación sucesiva del concepto, cuyas fuentes y formas llegan a incluir no sólo las relaciones de confianza establecidas dentro de pequeños grupos, sino también la efectividad de las normas estatales, la eficiencia burocrática, el parentesco, los medios de comunicación y las vías de tránsito, la seguridad pública, la religión y muchos etcéteras, tiene efectos diferentes de los esperados: en lugar de brindar elementos para clarificar el análisis y la discriminación de elementos diversos, oscurece la distinción entre la acción del estado, la 
de los municipios y autoridades locales, de los particulares, de los familiares y del mercado. Como en cajón de sastre, todo parece tener cabida en la teoría: empleo, religión, barrio, familia, gobierno, carreteras. Hasta el estado y el mercado, polos antagónicos alrededor de los cuales gravitaron las propuestas de política económica en los últimos siglos, conviven ahora, dentro de una noción que tal vez deba a su increíble ambigüedad y polisemia, las razones de su indudable poder de seducción. El CS, es, en efecto, un oscuro objeto.

En definitiva, a pesar de su aparente atractivo y de su indudable éxito, la nueva teoría del CS no constituye un aporte original ni un descubrimiento de los mecanismos de organización y funcionamiento de la vida social. La sociedad, tal como la misma teoría no duda en mostrar, tiende espontáneamente, a reproducirse a sí misma, a sus desigualdades y a sus mecanismos de atracción y de expulsión, lo que tiene lugar principalmente en el seno de los pequeños micro grupos donde procesa su reproducción biológica y donde se desarrolla la sociabilidad. Todavía fuertemente influida por el enorme peso de los supuestos individualistas de la teoría económica neoclásica, y habiendo olvidado los fundamentos universalistas y los propósitos igualitaristas que legitiman a la acción del estado moderno y de las políticas públicas, la teoría pretende encontrar el camino hacia la sociedad ideal por la vía de celebrar la realidad tal cual es, reivindicando su carácter moral y legitimando, con ello, un orden social particularista y desigual. 


\section{Referências Bibliogáficas}

ARROW, Kenneth - "Observations on social capital". In DASGUPTA, Partha; SERAGELDIN, Ismail (eds.). Social Capital. A Multifaceted Perspective, Washington DC: The World Bank, 2000, pp. 3-5.

BID - Reducción de la pobreza y fortalecimiento del capital social y la participación. La acción reciente del Banco Interamericano del desarrollo. Conferencia Regional "Capital Social y Pobreza". Santiago de Chile: CEPAL, 2001.

BOURDIEU, Pierre - "Las formas del capital. Capital Económico, capital cultural y capital social". In Idem. Poder, derecho y clases sociales. Barcelona: Desclée, 2000.

CHAVEZ, Daniel (ed.) - Más allá del mercado. El futuro de los servicios públicos, Ámsterdam: TNI, 2006, pp.9-22.

COLEMAN, James S. - "Social capital in the creation of human capital". In DASGUPTA, Partha; SERAGELDIN, Ismail (eds.) Social Capital. A Multifaceted Perspective, Washington DC: The World Bank, 2000.

FUKUYAMA, Francis - Social Capital, The Tanner Lectures On Human Values. Oxford: Brasenose College, 1997.

FONTES, Breno - "Terceiro Setor, Dádiva e Redes Sociais". In Medeiros, Alzira; Martins, Paulo Henrique (Org.) Economía Popular e Solidária. Desafios Teóricos y Práticos. Recife: Bagaço, 2003.

GRANOVETTER, Mark - "Economic action and social structure: the problem of embeddedness". American Journal of Sociology, 1985, pp. 481-510.

HELIWELL, John; PUTNAM, Robert - "Economic Growth and Social Capital in Italy". In DASGUPTA, Partha; SERAGELDIN, Ismail (eds.). Social Capital. A Multifaceted Perspective, Washington DC: The World Bank, 2000, pp. 253-269.

KLIKSBERG, Bernardo; TOMASSINI, Luciano - Capital social y cultura: Claves estratégcas para el desarrollo. Mexico: BID-FCE, 2000.

MARRERO, Adriana - "La teoría del Capital Social y la Educación en Uruguay". Actas del Congreso de Sociología de la Educación. Buenos Aires: ISA, 2004.

MARRERO, Adriana - "La teoría del capital social. Una crítica en perspectiva lationamericana". Arxius. no 14, 2006, pp. 73-90.

MARTINS, Paulo Henrique - "O Estado e a redistribuiçao dos bens da cidadania no contexto pós-nacional", In MARTINS, Paulo Henrique; FERREIRA NUNES, Brasilmar (Org). A nova ordem social. Perspectivas da solidaridades contemporanea, Brasília: Paralelo 15, 2004.

MEDEIROS, Alzira; MARTINS, Paulo Henrique (Org.) - Economía Popular e Solidária. Desafios Teóricos y Práticos. Recife: Bagaço, 2003.

MCDONALD, David; RUITERS, Greg - "Teorizando la privatización: contribución al desarrollo de una perspectiva de investigación crítica", In CHAVEZ, Daniel, (ed.) Más allá del mercado. El futuro de los servicios públicos, Ámsterdam: TNI, 2006, pp.9-22.

MCKINNEY, John C. - Tipología constructiva y teoría social. Buenos Aires: Amorrortu, 1968.

PORTES, Alejandro - "Social Capital. Its Origins and Applications in Modern Sociology". Annual Reviews. 1998, pp. 1-24.

PORTUGAL, Sílvia - "Contributos para uma discussão do conceito de rede na teoria sociológica", Oficina do CES, no 271, 2007.

PUTNAM, Robert - Per a fer que la democràcia funcioni. La importància del capital social. Barcelona: Proa, 2000.

SOLOW, R. "Notes on Social Capital and economic performance". In DASGUPTA, Partha; SERAGELDIN, Ismail (eds.). Social Capital. A Multifaceted Perspective. Washington DC: The World Bank, 2000, pp. 6-13.

TRIGLIA, C. - "Capital social y desarrollo local". In BAGNASCO, Arnaldo et al.. El capital social. Instrucciones de uso. Buenos Aires: FCE, 2003.

VIZER, Eduardo - La trama (in)visible de la vida social. Buenos Aires: La Crujía, 2006. 